\title{
Efecto del ayuno ante mortem en el rendimiento de la canal y calidad de la carne de conejo
}

\author{
Cornejo-Espinoza, J.G. ' ; Rodríguez-Ortega, L.T. '; Pro-Martínez, A. ${ }^{\circledR}$; González-Cerón, F. ${ }^{\text {; }}$ Conde-Martínez, V.F. ${ }^{\text {; }}$ \\ Ramírez-Guzmán, M.E. '; López-Pérez, E. ${ }^{2}$ y Hernández-Cázares, A.S. ${ }^{3}$
}

\author{
'Colegio de Postgraduados, Campus Montecillo. México. \\ ${ }^{2}$ Departamento de Zootecnia, Universidad Autónoma Chapingo. México. \\ ${ }^{3}$ Colegio de Postgraduados, Campus Córdoba. México.
}

\section{Palabras ClaVe adicionales \\ Rendimiento de la canal. \\ Capacidad de retención de agua. \\ Color. \\ pH.}

\section{RESUMEN}

El efecto del ayuno ante-mortem en la calidad de carne de conejo ha sido poco estudiado. Se utilizaron 180 conejos machos de la raza Nueva Zelanda Blanca de 2,0 kg $\pm 0,04$ de peso vivo (PV), los cuales fueron distribuidos en cuatro tratamientos $(0,4,8$ y $12 \mathrm{~h}$ de ayuno). Se utilizó un diseño completamente al azar, con el peso vivo al sacrificio como covariable. Se evaluaron las pérdidas de peso vivo (PV), el rendimiento de la canal, el pH inicial de la canal y final $(24 \mathrm{~h})$ de la carne. En el músculo Longissimus dorsi se evaluó el color, la capacidad de retención de agua (CRA), las pérdidas de cocción y la fuerza de corte. El PV disminuyó $0,31,54$ y $76 \mathrm{~g}$ respecto al peso inicial, según el orden de los tratamientos. El rendimiento de la canal con $12 \mathrm{~h}$ de ayuno aumentó con respecto al testigo. El pH inicial fue mayor con $8 \mathrm{~h}$ respecto al resto de los tratamientos; sin embargo, el pH final fue menor con $12 \mathrm{~h}$ de ayuno con respecto al testigo. El color no se modificó por efecto de los tratamientos. La CRA disminuyó con 4 y $8 \mathrm{~h}$ de ayuno y la fuerza de corte se redujo con $8 \mathrm{~h}$ de ayuno, ambas respecto al testigo. Cuando se incrementó el tiempo de ayuno se reduce el peso de la víscera digestiva y urinaria e incrementa el rendimiento de la canal.

\section{Effect of ante-mortem fasting on carcass yield and quality of rabbit meat}

\section{SUMMARY}

The effect of ante-mortem fasting on the quality of rabbit meat has been little studied. One hundred and eighty male rabbits of New Zealand White breed of $2.0 \pm 0.04 \mathrm{~kg}$ of body weight (BW) were used and distributed in four treatments $(0,4,8$ and $12 \mathrm{~h}$ of fasting). A completely randomized design was used, with live weight at slaughter as a covariate. Body weight losses, carcass yield, the initial and final $\mathrm{pH}(24 \mathrm{~h})$ of meat were evaluated. Color, water holding capacity (WHC), cooking losses and the shear force were evaluated in Longissimus dorsi muscle. The BW decreased 0, 31, 54 and $76 \mathrm{~g}$ with respect to the initial body weight, in the order of the treatments. Carcass yield increased with $12 \mathrm{~h}$ of fasting, compared to the control group. The initial $\mathrm{pH}$ was greater at $8 \mathrm{~h}$ regarding other treatments; however, the final $\mathrm{pH}$ was lower at $12 \mathrm{~h}$ of fasting compared with the control. Color was not modified by the effect of treatments. WHC decreased at 4 and $8 \mathrm{~h}$ of fasting and shear force decreased at $8 \mathrm{~h}$ of fasting, both with respect to the control. When the fasting time increased, the weight of digestive and urinary viscera decreased and carcass yield increased.

\section{INFORMACIÓN}

Cronología del artículo.

Recibido/Received: 10.04.2015

Aceptado/Accepted: 02.03.2016

On-line: 11.06 .2016

Correspondencia a los autores/Contact e-mail:

aproma@colpos.mx

\section{INTRODUCCIÓN}

La producción de carne de conejo en México es una actividad agropecuaria basada principalmente en sistemas de producción extensivo o de traspatio (características que demandan pocos insumos, baja producción y poca tecnología,) y con poco impacto los sistemas semi-intensivo e intensivo (este último con buenas instalaciones y tecnología) (Olivares et al., 2009). Esta situación, origina un limitado desarrollo de infraestructura: como mataderos, empacadoras e industrializadoras. Lo que obliga a los productores a sacrificar los conejos en la misma granja o a desplazarlos grandes distancias (Jolley, 1990). Estas condiciones de manejo pueden modificar la conversión de músculo a carne, por efecto del estrés previo al proceso de matanza, principalmente características como: la cantidad de agua retenida, las pérdidas por cocción, la fuerza de corte, el rendimiento de la canal y el pH (María et al., 2006; Liste et al., 2009), y características sensoriales 
como color, sabor y textura (Liste et al., 2009). El cambio del $\mathrm{pH}$ de la carne a las $24 \mathrm{~h}$ es muy importante ya que puede disminuir el rendimiento de la canal o la apariencia, provocando rechazo de ésta por el consumidor (Hernández, 1997).

El estrés ante mortem tiene efectos negativos en la calidad de la carne de conejo (Liste et al., 2008; María et al., 2008; Mačanga et al., 2011). En México la Norma Oficial NOM-033-ZOO-1995 no contempla recomendaciones sobre el tiempo de ayuno o de transporte. Hambrecht et al. (2005) mencionan que el estrés ante mortem es el principal factor responsable de la reducción de la calidad de la carne de cerdo.

El ayuno mediante el retiro del agua y el alimento a los conejos antes de la matanza disminuye problemas de contaminación de la canal por la excreción de heces y orina durante el eviscerado (Kola et al., 1994). Borel et al. (2001) mencionan que es necesario después de ese periodo de estrés, dar a los animales un periodo de relajación, para que el animal recupere la homeostasis; es decir, se recobren las concentraciones de glucógeno. Zhang et al. (2009) señalan que en pollos de engorda un largo periodo de recuperación después del transporte disminuyó los niveles de cortisol en plasma y la glucolisis del músculo, lo cual puede mejorar la calidad de la carne, pero en conejos aún no está determinado con certeza. El objetivo de esta investigación fue evaluar el efecto de cuatro tiempos de ayuno ante mortem en la pérdida de peso vivo, pérdida de peso en la víscera digestiva y urinaria llena y vacía, rendimiento de la canal, $\mathrm{pH}$ inicial en la canal y final en la carne y la capacidad de retención de agua y fuerza de corte en el músculo Longissimus dorsi.

\section{MATERIALES Y MÉTODOS}

Se utilizaron 180 conejos machos de la línea Nueva Zelanda Blanca, con 2,0 kg de PV. Dos días previos a la matanza, se alojaron en jaulas individuales de 40x70x40 cm. Recibieron alimento comercial y se asignaron aleatoriamente a cuatro tratamientos de 45 conejos cada uno. Estos consistieron en retirar el alimento y el agua a 0 (testigo), 4, 8 y 12 h previas a la matanza; ésta se hizo con base en la NOM-033-ZOO-1995.

Para determinar las pérdidas de PV se registró el peso de cada animal antes de someter los animales a los tratamientos y antes de la matanza. Durante los primeros 15 min después de la matanza y una vez retirada la piel, se midió el $\mathrm{pH}$ inicial de la canal $\left(\mathrm{pH}_{\mathrm{i}}\right)$ (Lambertini et al., 2006) con un medidor de $\mathrm{pH}$ portátil (HANNA INS. INC. Mod. HI 99161. Rhode Island, USA.), provisto de un electrodo de punción, el cual se colocó entre la sexta y séptima vértebra lumbar del lado derecho de la canal a una profundidad de $1,5 \mathrm{~cm}$. Las canales se mantuvieron por 30 min en un área ventilada a temperatura ambiente (Pascual y Pla, 2007), y posteriormente se mantuvieron en refrigeración a $4^{\circ} \mathrm{C}$, hasta el registro del $\mathrm{pH}$ final de la carne $\left(\mathrm{pH}_{\mathrm{f}}\right)$ a las 24 h post-mortem, en el mismo lugar anatómico en el que se registró el $\mathrm{pH}_{\mathrm{i}}$. El peso de la víscera torácica (pulmones, corazón, y tráquea) y la canal caliente se registraron con base a la metodología propuesta por Blasco et al. (1992). El hígado se pesó por separado de la canal sin considerar la vesícula. También, se registró el peso de la víscera digestiva y urinaria llena (PVDULL) y vacía (PVDUV), expresado como porcentaje del peso vivo a la matanza, las que comprenden el estómago, intestino, tracto urogenital y la vejiga (de Blas, 1992; Blasco y Ouhayoun, 1993). El cálculo del peso del contenido digestivo y urinario (PCDU) fue con la ecuación PCDU=PVDULL-PVDUV. Las pérdidas por cocción (PCC) se determinaron por diferencia de peso del lomo crudo y cocido. Para ello, el lomo se pesó y se colocó en una bolsa de polietileno de 12 × $20 \mathrm{~cm}$, en un baño de agua a $75{ }^{\circ} \mathrm{C}$ hasta alcanzar una temperatura interna de $70^{\circ} \mathrm{C}$ (María et al., 2006), después se extrajo el lomo de la bolsa y se mantuvo $15 \mathrm{~min}$ a temperatura ambiente, se pesó y se calculó las PCC mediante la siguiente ecuación [PCC $=(\mathrm{Pi}-\mathrm{Pf}) \times 100 / \mathrm{Pi}]$, donde $\mathrm{Pi}$ es el peso antes de la cocción y Pf es el peso después de la cocción. Los lomos se mantuvieron en refrigeración envueltos en papel aluminio por otras $24 \mathrm{~h}$ para determinar la fuerza de corte. La fuerza de corte se determinó 48 h post-mortem en un texturómetro (Modelo EZ-SX short, Dübendorf, Suiza) mediante la prueba de Warner Bratzler. Se utilizó el músculo Longissimus dorsi cocido de la prueba de PCC, el cual se dividió en tres porciones cortadas perpendicularmente a las fibras musculares de $2 \mathrm{~cm}$ de largo $x 1 \mathrm{~cm}$ de ancho $x 1 \mathrm{~cm}$ de espesor, con una velocidad de corte de $10 \mathrm{~mm} / \mathrm{s}$.

La medición del color (Luminosidad, $L^{*}$; rojo, $a^{*}$; y amarillo, $\left.b^{*}\right)$ se analizó en un corte transversal de 10 $\mathrm{mm}$ de espesor en la parte media del músculo Longissimus dorsi a las $24 \mathrm{~h}$ post-mortem (Boccard et al., 1981; Pascual y Pla, 2007), con un colorímetro (Minolta CR-400/410, Tokio, Japón); calculando los índices de Croma $\left(C^{*}\right)$ y hue $\left(h^{*}\right)$ mediante las siguientes ecuaciones: $C^{*}=\left(\left(a^{*}\right)^{2}+\left(b^{*}\right)^{2}\right)^{1 / 2}$ y $h^{*}=\tan ^{-1}\left(b^{*} / a^{*}\right)$ (María et al., 2006).

La capacidad de retención de agua (CRA) se determinó utilizando el método propuesto por Guerrero et al. (2002). Una muestra de 2,5 g de lomo finamente picada se homogeneizó con una varilla de vidrio por un minuto en $4 \mathrm{~mL}$ de una solución de cloruro de sodio (Marca Mollinckrodt AR. USA) 0,6 M y se dejó reposar por 30 min en un baño de hielo. Después se centrifugó a 10,000 r.p.m durante 15 min, el sobrenadante se midió en una probeta y representa el agua no retenida.

Los datos se analizaron con el programa SAS 9.0 (2002) después de haber comprobado la normalidad de los datos por una prueba de BOX-COX y una prueba Wald-Wolfowitz para los residuales, con un modelo estadístico completamente al azar y un procedimiento PROC MIXED, que incluyó efectos fijos (tratamientos y fecha de matanza) y efectos aleatorios (animal). Además, se utilizó el PV a la matanza como covariable.

\section{RESULTADOS Y DISCUSIÓN}

La cadena de producción de la carne finaliza con el manejo que reciben los conejos previo a la matanza, el cual puede poner en riesgo el trabajo de los productores durante el periodo de engorda (María et al., 2008).

El peso de los conejos no fue significativo $(p>0,05)$; sin embargo, las pérdidas de PV difirieron con $4 \mathrm{y}$ 
$12 \mathrm{~h}$ de ayuno con respecto al testigo. Estas pérdidas representaron 1,5, 2,7 y 3,7\% del PV previo a los tratamientos de 4,8 y 12 h de ayuno respectivamente (tabla I). Jolley (1990) menciona que las pérdidas de peso en conejos ayunados por 12 y $24 \mathrm{~h}$ antes del transporte oscilan entre 3 y 5\%, respectivamente. De Blas (1992) y Ashby et al. (1980) mencionan que en periodos cortos de ayuno (12 h) las pérdidas de PV oscilan entre 3-4\%, concordando con las pérdidas de PV encontradas con $12 \mathrm{~h}$ de ayuno en este estudio (tabla I). Las pérdidas de PV durante un periodo de ayuno se deben a la excreción de heces y orina (Kirton et al., 1968), lo que puede tener un efecto positivo en la calidad de la canal (Margüenda et al., 2008) ya que disminuye la probabilidad de que durante el eviscerado se contamine con heces y orina (Komiyama et al., 2008). Sin embargo, cuando los conejos son sometidos a estrés calórico a una temperatura de $42^{\circ} \mathrm{C}$ durante una hora, las pérdidas de peso son de aproximadamente $20 \%$ (Amici et al., 2000) no recuperándose hasta el quinto día, tiempo de adaptación requerido a estas condiciones, lo que sin duda puede resultar en pérdidas económicas en regiones climáticas con altas temperaturas. En regiones de México como: Sonora, Sinaloa, Veracruz y Tabasco se alcanza temperaturas superiores a los $40^{\circ} \mathrm{C}$ en ciertos meses del año. Sin embargo, la temperatura en Texcoco, México en julio varió de 19 a $23^{\circ} \mathrm{C}$ (CONAGUA, 2014).

La importancia del contenido digestivo y urinario está relacionada con la calidad de la canal ya que afecta directamente el rendimiento final de la cadena de producción (De Blas, 1992). El PVDULL disminuyó con 8 y 12 h de ayuno con respecto al testigo (tabla I), esto confirma la pérdida de PV a la matanza, ya que PVDUV no cambió por efecto de tratamientos como lo han citado Coppings et al. (1989).

El rendimiento de la canal es el criterio comercial económico más importante en España en el mercado del conejo, y los resultados de esta variable en el presente estudio fueron similares a los de Pla et al. (1998). La duración del ayuno modificó el rendimiento de la canal debido a la disminución del PCDU con los tiempos más largos de ayuno (tabla I), esto concuerda con los datos de otros autores (Jolley, 1990; Masoero et al., 1992; Ouhayoun y Dalle Zotte, 1993; Lambertini et al., 2006). Los rendimientos más altos fueron en los conejos con 8 y 12 h de ayuno con respecto al testigo $(0 \mathrm{~h})$, concordando con Margüenda et al. (2008) en conejos con $12 \mathrm{~h}$ de ayuno. La restricción de alimento ocasiona una adaptación metabólica en los animales para cubrir los requerimientos de energía, esto provoca un gasto energético que afecta el glucógeno almacenado en hígado y músculo, por lo que a mayor tiempo de ayuno menor será la concentración de glucógeno en esos tejidos (Masoero et al., 1992). El hecho de que el conejo dependa de sus reservas energéticas puede ser un problema de importancia económica, ya que los conejos con hambre suelen ser más nerviosos que los alimentados ad libitum (Masoero et al., 1992), ocasionando que se agoten las reservas energéticas necesarias para el proceso post mortem de la carne, lo que afecta características como el pH, la CRA, el color y la dureza. Los resultados de este estudio mostraron disminución del peso del hígado cuando aumenta el tiempo de ayuno (tabla I); Cop-
Tabla I. Medias de mínimos cuadrados de los parámetros físicos sujetos a diferentes tiempos de ayuno (Least squares means of physical parameters subject to different times of fasting).

\begin{tabular}{|c|c|c|c|c|c|}
\hline \multirow{2}{*}{ Variables } & \multicolumn{4}{|c|}{ Tiempo de ayuno (h) } & \multirow{2}{*}{ E.E } \\
\hline & 0 & 4 & 8 & 12 & \\
\hline $\begin{array}{l}\text { PV previo al } \\
\text { tratamiento }(\mathrm{g})\end{array}$ & 2000 & 2080 & 2020 & 2050 & 38 \\
\hline PV sacrificio (g) & 2034 & 2049 & 1966 & 1974 & 36 \\
\hline Pérdidas PV (g) & $0^{\mathrm{b}}$ & $31^{\mathrm{b}}$ & $54^{\mathrm{ab}}$ & $76^{a}$ & 0,3 \\
\hline Perdidas $(\%)^{*}$ & $0^{c}$ & $1,5^{\mathrm{b}}$ & $2,7^{\mathrm{ab}}$ & $3,7^{\mathrm{a}}$ & 0,2 \\
\hline \multicolumn{6}{|c|}{ Víscera digestiva y urinaria } \\
\hline PVDULL (g) & $448^{a}$ & $416^{\mathrm{ab}}$ & $378^{b}$ & $383^{b}$ & 36 \\
\hline $\operatorname{VDULL}(\%)^{\dagger}$ & $22,0^{\mathrm{a}}$ & $20,3^{\mathrm{ab}}$ & $19,2^{b}$ & $19,4^{b}$ & 0,48 \\
\hline PVDUV (g) & 140 & 137 & 132 & 134 & 11 \\
\hline $\operatorname{VDUV}(\%)^{\dagger}$ & 6,9 & 6,7 & 6,7 & 6,8 & 0,18 \\
\hline PCDU (g) & $308^{a}$ & $279^{\mathrm{ab}}$ & $246^{b}$ & $249^{b}$ & 33 \\
\hline $\mathrm{CDU}(\%)^{\dagger}$ & $15,09^{a}$ & $13,61^{b}$ & $12,56^{b}$ & $12,61^{b}$ & 0,49 \\
\hline $\begin{array}{l}\text { Rendimiento en } \\
\text { canal }(\%)^{\dagger}\end{array}$ & $54,5^{b}$ & $55,6^{a b}$ & $56,7^{a}$ & $57,6^{a}$ & 0,8 \\
\hline $\begin{array}{l}\text { Víscera torácica } \\
(\%)^{\dagger}\end{array}$ & 1,08 & 1,19 & 1,25 & 1,07 & 0,14 \\
\hline Hígado $(\%)^{\dagger}$ & $2,8^{\mathrm{a}}$ & $2,8^{a}$ & $2,5^{\mathrm{b}}$ & $2,5^{\mathrm{b}}$ & 0,09 \\
\hline
\end{tabular}

${ }^{\dagger}$ como \% del PV al sacrificio; *como \% del PV previo al tratamiento; PVDULL=Peso de la víscera digestiva y urinaria llena; VDULL=Víscera digestiva y urinaria llena; PVDUV=Peso de la víscera digestiva y urinaria vacía; VDUV= Víscera digestiva $\mathrm{y}$ urinaria vacía; PCDU=Peso del contenido digestivo y urinario; $\mathrm{CDU}=$ Contenido digestivo y urinario; literales ${ }^{\mathrm{a}, \mathrm{b}, \mathrm{c}}$ diferentes representan diferencias significativas $p<0,05$.

pings et al. (1989) mencionan que las pérdidas de peso del hígado pueden atribuirse a una deshidratación de la víscera en general, esta es una posible explicación a los resultados observados en esta investigación, pues los conejos con 8 y $12 \mathrm{~h}$ de ayuno tuvieron el menor peso de hígado comparados con el testigo.

Poca información se conoce en relación al color de la carne de conejo con ayuno ante-mortem (Liste et al., 2009). Ouhayoun y Dalle Zotte (1993) indican que la concentración de pigmentos en la carne de conejo varía por el metabolismo de la energía, donde la actividad anaeróbica de los músculos aumenta con la edad provocando cambios en el pH del músculo que a su vez modifica la estabilidad del color; y por la edad se acumulan más pigmentos debido a que las dietas de conejos incluyen altas cantidades de forrajes verdes. En bovinos hay estudios que indican que el color de la carne depende del grado de oxidación de la mioglobina en el músculo (Renerre, 1990) que se modifica por el manejo previo al sacrificio como el transporte, ayuno o temperatura ambiental (Hambrecht et al., 2005; Lambertini et al., 2006). En este sentido, la coloración de la carne de conejo a las $24 \mathrm{~h}$ post-mortem, no cambió con el tiempo de ayuno (tabla II). En un estudio realizado por 
Tabla II. Medias de mínimos cuadrados de las variables de calidad $24 \mathrm{~h}$ post-mortem en carne de conejos sometidos a diferentes tiempos de ayuno (Least squares means of quality variables $24 \mathrm{~h}$ post-mortem in meat rabbits at different times of fasting)

\begin{tabular}{|c|c|c|c|c|c|}
\hline \multirow{2}{*}{ Variables $_{24 \mathrm{~h}}$} & \multicolumn{4}{|c|}{ Tiempo de ayuno (h) } & \multirow{2}{*}{ E.E. } \\
\hline & 0 & 4 & 8 & 12 & \\
\hline $\mathrm{PCF}(\mathrm{g})$ & 1128,2 & 1138,71 & 1133,8 & 1163,48 & 14,6 \\
\hline \multicolumn{6}{|c|}{ Color } \\
\hline$L^{*}$ & 62,19 & 61,72 & 62,70 & 62,52 & 0,4 \\
\hline$a^{*}$ & 12,02 & 12,58 & 12,41 & 12,01 & 0,3 \\
\hline$b^{*}$ & 4,98 & 5,05 & 5,34 & 4,93 & 0,2 \\
\hline$C^{*}$ & 12,99 & 13,49 & 13,46 & 12,95 & 0,33 \\
\hline$h^{*}$ & 2,55 & 2,55 & 2,27 & 2,56 & 0,14 \\
\hline $\mathrm{pH}_{\mathrm{i}}$ & $6,06^{b}$ & $6,14^{b}$ & $6,32^{a}$ & $6,15^{b}$ & 0,04 \\
\hline $\mathrm{pH}_{\mathrm{f}}$ & $5,74^{a}$ & $5,69^{a b}$ & $5,70^{\mathrm{ab}}$ & $5,65^{b}$ & 0,02 \\
\hline $\mathrm{CRA}\left(\mathrm{ml} 100 \mathrm{~g}^{-1}\right)$ & $37,3^{a}$ & $28,4^{b}$ & $22,8^{b}$ & $30,2^{\mathrm{ab}}$ & 2,7 \\
\hline $\operatorname{PCC}\left(\mathrm{ml} 100 \mathrm{~g}^{-1}\right)$ & 15,6 & 13,8 & 13,0 & 12,3 & 1,9 \\
\hline $\begin{array}{l}\text { Fuerza de corte } \\
\left(\mathrm{g} / \mathrm{cm}^{2}\right)\end{array}$ & $987,7^{a}$ & $969,9^{a}$ & $699^{b}$ & $809,9^{a b}$ & 91,1 \\
\hline
\end{tabular}

$\mathrm{PCF}=$ peso de la canal fría; $\mathrm{pH}_{\mathrm{i}}=\mathrm{pH}$ inicial; $\mathrm{pH}_{\mathrm{f}}=\mathrm{pH}$ final; $\mathrm{CRA}=$ capacidad de retención de agua; $\mathrm{PCC}=$ pérdidas por cocción; medias con literales ${ }^{a, b}, \mathrm{c}$ diferentes representan diferencias significativas $p<0,05$.

Lambertini et al. (2006) donde evaluaron el tiempo y la densidad de población durante el transporte reportan valores de $L^{*}$ muy parecidos a los encontrados en este estudio. Los resultados de Luminosidad de esta investigación no mostraron diferencias en $L^{*}$. Sin embargo, Jolley (1990) reporta que cuando se incrementa las horas de ayuno a $24 \mathrm{~h}$ la $L^{*}$ disminuye. Los valores de hue $\left(h^{*}\right)$ y croma $\left(C^{*}\right)$ mostraron el mismo comportamiento que las variables $a^{*}, b^{*}$ y $L^{*}$ (tabla II).

En este estudio, respecto a los cambios físico-químicos posteriores al rigor mortis de la carne, el $\mathrm{pH}$ es una de las principales variables que se utiliza para medir y clasificar la calidad de la misma (María et al., 2006; Liste et al., 2009), ya que la luminosidad de la carne, la capacidad de retención de agua y la dureza dependen del valor de $\mathrm{pH}$ de la carne (Ouhayoun y Dalle Zotte, 1993). Las condiciones peri-mortem (ayuno, transporte, enfriado de la canal, etc.) ocasionan un desequilibrio en el metabolismo de la energía disminuyendo el $\mathrm{pH}$ más rápido (Ouhayoun y Dalle Zotte, 1993; María et al., 2008).

Después de la matanza ocurren cambios bioquímicos en el tejido muscular como la disminución de $\mathrm{pH}$ (Ouhayoun y Dalle Zotte, 1993). Esta afirmación concuerda con los resultados de esta investigación, pues el menor $\mathrm{pH}_{\mathrm{f}}$ en la carne se observó con $12 \mathrm{~h}$ de ayuno (tabla II). Liste et al. (2009) mencionan que los periodos cortos de estrés ocasionan que se incremente el movimiento de reservas de energía dado que los conejos tienen que adaptarse a nuevas circunstancias, en este caso al tiempo de ayuno. Alarcón et al. (2005) demos- traron que en cerdos la disminución del $\mathrm{pH}_{\mathrm{i}}$ se atribuye a largos periodos de espera al sacrificio al aumentar el movimiento por peleas en los corrales, disminuyendo las reservas de glucógeno. Sin embargo, los resultados de esta investigación no concuerdan con los observados por Margüienda et al. (2008) quienes evaluaron $12 \mathrm{y}$ $24 \mathrm{~h}$ de ayuno en conejos y no encontraron diferencias en el pH de las canales 24 h post mortem. Pla et al. (1998) señalan que el pH del Longissimus dorsi es menor al del Biceps femoris, estos resultados difieren ligeramente con los reportados en el presente estudio para el músculo Longissimus dorsi.

El $\mathrm{pH}_{\mathrm{f}}$ que se obtuvo en los animales con $12 \mathrm{~h}$ de ayuno (tabla II) podría sugerir que la carne fuera PSE (pálida, suave, exudativa). Alarcón et al. (2005) señalan que en cerdos, valores de $\mathrm{pH}$ inferiores de 5,9 corresponderían a una carne PSE. De acuerdo con los valores obtenidos en este estudio con todos los tratamientos evaluados se obtuvo ese tipo de carne.

La CRA disminuyó en los tratamientos de 4 y 8 h de ayuno con respecto al testigo (tabla II). Dalle Zotte et al. (1995) señalan que las pérdidas de agua en la carne aumentan a medida que disminuye el $\mathrm{pH}$ final. Los resultados de esta investigación no concuerdan con la afirmación anterior, puesto que el menor $\mathrm{pH}_{\mathrm{f}}$ se observó con 12 h de ayuno.

La dureza o suavidad de la carne está relacionada con la cantidad y calidad de colágeno en la carne; sin embargo, como los conejos fueron sacrificados muy jóvenes (70 días de edad), la cantidad de colágeno no es un impedimento para deducir que este sea un factor que influya en la determinación de la dureza de la carne. En conejos se ha encontrado que a pesar de su alta sensibilidad a la desnaturalización proteica durante la maduración suele ocurrir un aumento en la dureza de la carne (Ouhayoun y Dalle Zotte, 1993).

Para determinar la fuerza de corte, la navaja Warner Bratzler es un buen predictor de dureza en carne cocida, que es considerado como criterio para la aceptabilidad por los consumidores (Liste et al., 2009; Combes et al., 2003). Lambertini et al., (2006) mencionan que el transporte influye sobre la dureza de la carne y el $\mathrm{pH}_{\mathrm{f}}$. María et al. (2008) mencionan que pequeñas variaciones en el $\mathrm{pH}$ pueden afectar la dureza de la fibra, atribuido a un acortamiento del sarcómero.

En este estudio se observó que con 8 h de ayuno la fuerza de corte y la capacidad de retención de agua disminuyeron con respecto al testigo (tabla II). Estos resultados no coinciden con los reportados por Trocino et al., (2003) quienes al evaluar hasta $8 \mathrm{~h}$ de transporte no encontraron diferencias en las perdidas por cocción y en la fuerza de corte. Respecto al $\mathrm{pH}_{\mathrm{f}}$ a $12 \mathrm{~h}$ de ayuno estos valores difirieron a los de Jolley (1990) con $24 \mathrm{~h}$.

\section{CONCLUSIÓN}

A medida que incrementa el tiempo de ayuno en los conejos se reduce el peso de la víscera digestiva y urinaria llena e incrementa el rendimiento de la canal. La fuerza de corte y capacidad de retención de agua en el músculo Longisimus dorsi se reduce con $8 \mathrm{~h}$ de ayuno con respecto al testigo. 


\section{AGRADECIMIENTOS}

Al Consejo Nacional de Ciencia y Tecnología (CONACYT), Colegio de Postgraduados, también a la Línea Prioritaria de Investigación 12, por el financiamiento y facilidades otorgadas para el término de este estudio.

\section{BIBLIOGRAFÍA}

Alarcón, R.A.D.; Duarte, A.J.O.; Rodríguez, A.F.A. and Janacua, V.H. 2005. Incidence of PSE and DFD muscle in pigs slaughtered in Mexico's Bajio region. Téc Pecu Méx, 43: 335-346.

Amici, A.; Franci, O.; Mastroiacono, P.; Merendino, N.; Nardini, M. and Tomassi, G. 2000. Short term acute heat stress in rabbits: functional, metabolic and immunological effects. World Rabbit Sci, 8: 11-16.

Ashby, B.H.; Ota, H.; Bailey, A.; Whitehead, J.A. and Kindya, W.G. 1980. Heat and weight loss of rabbits during simulated air transport. Trans ASAE, 23: 162-164.

Blasco, A.; Ouhayoun, J. and Masoero, G. 1992. Study of rabbit meat and carcass. J Appl Rabbit Res, 15: 775-776.

Blasco, A. and Ouhayoun, J. 1993. Harmonization of criteria and terminology in rabbit meat research. World Rabbit Sci, 4: 93-99.

Boccard, R.; Buchter, L.; Casteels, E.; Cosentino, E.; Dransfield, E.; Hood, D.E.; Joseph, R.L.; Macdougall, D.B.; Rhodes, D.N.; Schon, I.; Tinbergen, B.J. and Touraille, C. 1981. Procedures for measuring meat quality characteristics in beef production experiments. Report of a working group in the commission of the European communities (CEC) beef production research programme. Livest Prod Sci, 8: 385-397.

Borel, V.E.H. 2001. The biology of stress and its application to livestock housing and transportation assessment. J Anim Sci, 79: E26-E260.

Combes, S.; Lepetit, J.; Darche, B. and Lebas, F. 2003. Effect of cooking temperature and cooking time on Warner-Bratzler tenderness measurement and collagen content in rabbit meat. Meat Sci, 66: 91-96.

CONAGUA (Comisión Nacional del Agua). 2014. Reporte del clima en México, reporte anual 2014. Editorial: Coordinación General del Servicio Meteorológico Nacional. Gerencia de Meteorología y Climatología. Subgerencia de Pronóstico a Mediano y Largo Plazo. México D.F. pp. 1-27.

Coppings, R.J.; Ekhator, N. and Ghodrati, A. 1989. Effects of antemortem treatment and transport on slaughter characteristics of fryer rabbits. J Anim Sci, 67: 872-880.

Dalle Zotte, A.; Parigi Bini, R.; Xiccato, G. and Simionato, S. 1995 Proprieta tecnologiche e sensoriali della carne di coniglio. Riv di coniglicoltura, 32: 33-39.

De Blas, C. 1992. Factores que determinan el rendimiento y la calidad de la canal en conejos. Mundo ganadero. Cunicultura, 7: 70-75.

Guerrero, L.I.; Ponce, A.E. y Pérez, M.L. 2002. Curso práctico de tecnología de carnes y pescado. Universidad Autónoma Metropolitana. Unidad Iztapalapa. México. D. F. 171 pp.

Hambrecht, E.; Eissen, J.J.; Newman, D.J.; Smits, C.H.M.; den Hartog, L.A. and Verstegen, M.W.A. 2005. Negative effects of stress immediately before slaughter on pork quality are aggravated by suboptimal transport and lairage conditions. J Anim Sci, 83: 440-448.

Hernández, P. 1997. Calidad de la carne de conejo. Lagomorpha, 90: $13-19$.

Jolley, P.D. 1990. Rabbit transport and its effects on meat quality. Appl Anim Behaviour Sci, 28: 119-134.

Kirton, A.H.; Quartermain, A.R.; Uliee, A.E.; Carter, W.A. and Pickering, F.S. 1968. Effect of 1 and 2 days' ante-mortem fasting on live weight and carcass losses in lambs. N Z J Agric Res, 11: 891-902.
Kola, J.; Awosanya, B. and Adebua, B.A. 1994. The effects of preslaughter whithholding of feed and water from rabbits on their carcass yield and meat quality. Nig J Anim Prod, 21: 164-169.

Komiyama, C.M.; Mendes, A.A.; Takahashi, S.E.; Moreira, J.; Garcia, R.G.; Sanfelice, C.; Borba, H.S.; Leonel, F.R.; Paz, I.C.L.A. and Balog, A. 2008. Chicken meat quality as a function of fasting period and water spray. Braz J Poultry Sci, 10: 179-183.

Lambertini, L.; Vignola, G.; Badiani, A.; Zaghini, G. and Formigoni, A. 2006. The effect of journey time and stocking density during transport on carcass and meat quality in rabbits. Meat Sci, 72: 641-646.

Liste, M. G.; María, G.A.; García-Belenguer, S.; Chacón, G.; Gazzola, P. and Villarroel, M. 2008. The effect of transport time, season and position on the truck on stress response in rabbits. World Rabbit Sci, 16: $229-235$

Liste, G.; Villarroel, M.; Chacón, G.; Sañudo, C.; Olleta, J.L.; GarcíaBelenguer, S.; Alierta, S. and María, G.A. 2009. Effect of lairage duration on rabbit welfare and meat quality. Meat Sci, 82: 71-76.

Mačanga, J.; Koréneková, B.; Nagy, J.; Marcinčák, S.; Popelka, P.; Kožárová, I. and Korének, M. 2011. Post-mortem changes in the concentration of lactic acid, phosphates and pH in the muscles of wild rabbits (Oryctolagus cuniculus) according to the perimortal situation. Meat Sci, 88: 701-704.

Margüenda, I.; Nicodemus, N.; García-Rebollar, P.; Villarroel, M.; Sevilla, L.; Vadillo, S. y Carbaño, R. 2008. Efecto del tiempo de ayuno sobre el rendimiento y la calidad microbiológica de la canal del conejo. Resúmenes del XXXIII Symposium de ASESCU. España. 24-27 pp.

María, G.A.; Buil, T.; Liste, G.; Villarroel, M.; Sañudo, C. and Olleta, J.L. 2006. Effects of transport time and season on aspects of rabbit meat quality. Meat Sci, 72: 773-777.

María, G.; Liste, G.; Campo, M.M.; Villarroel, M.; Sañudo, C.; Olleta, J.I. and Alierta, S. 2008. Influence of transport duration and season on sensory meat quality in rabbits. World Rabbit Sci, 16: 81-88.

Masoero, G; Riccion, L.; Bergoglio, G. and Napolitano F. 1992. Implications of fasting and of transportation for a high quality rabbit meat product. Proc. $5^{\text {th }}$ World Rabbit Congress, Corvallis. Oregon, USA. Volume B: 841-847

NOM-033-ZOO-1995. Sacrificio humanitario de los animales domésticos y silvestres. Servicio Nacional de Sanidad, Inocuidad y Calidad Agroalimentaria (SENASICA). Secretaría de Agricultura, Ganadería, Desarrollo Rural, Pesca y Alimentación (SAGARPA). Diario Oficial de la Federación. México D.F. México. 7 de julio de 1995. 12 pp.

Olivares, P.R.; Gémez, C.M.A.; Schwentesius, R.R.; Carrera, C.B. 2009. Alternativas a la producción y mercadeo para la carne de conejo en Tlaxcala, México. Región y Sociedad, XXI : 191-207.

Ouhayoun, J. and Dalle Zotte, A. 1993. Muscular energy metabolism and related traits in rabbit. A review. World Rabbit Sci, 1: 97-108.

Pascual, M. and Pla, M. 2007. Changes in carcass composition and meat quality when selecting rabbits for growth rate. Meat Sci, 77: 474-481.

Pla, M.; Guerrero, L.; Guardia, D.; Oliver, M.A. and Blasco, A. 1998. Carcass characteristics and meat quality of rabbit lines selected for different objectives: I. Between lines comparison. Livest Prod Sci, 54: 115-123.

Renerre, M. 1990. Review: Factors involved in the discoloration of beef meat. Int J Food Sci Tech, 25: 613-630.

SAS. 2002.Statistical Analysis System Institute. Cary, N.C. USA. 1028 pp. Trocino, A.; Xiccato, G.; Queaque, P.I. and Sartori, A. 2003. Effect of transport duration and gender on rabbit carcass and meat quality. World Rabbit Sci, 11: 23-32.

Zhang, L.; Yue, H.Y.; Zhang, H.J.; Xu, L.; Wu, S. G.; Yan, H.J.; Gong, Y.S. and Qi, G.H. 2009. Transport stress in broilers: I. Blood metabolism, glycolytic potential, and meat quality. Poult Sci, 88: 2033-2041. 